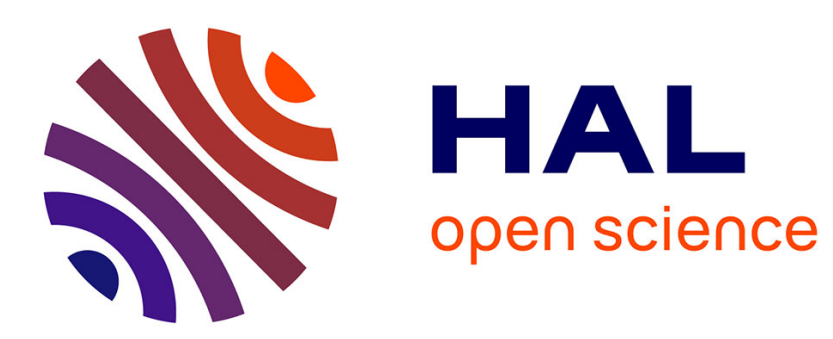

\title{
ALMA, A Logistic Mobile Application based on Internet of Things
}

Didier El Baz, Julien Bourgeois, Toufik Saadi, Alessandro Bassi

\section{To cite this version:}

Didier El Baz, Julien Bourgeois, Toufik Saadi, Alessandro Bassi. ALMA, A Logistic Mobile Application based on Internet of Things. 2013 IEEE International Conference on Internet of Things, Aug 2013, Pékin, China. pp.355-358, 10.1109/GreenCom-iThings-CPSCom.2013.78 . hal-00931515v2

\section{HAL Id: hal-00931515 \\ https://hal.science/hal-00931515v2}

Submitted on 19 May 2015

HAL is a multi-disciplinary open access archive for the deposit and dissemination of scientific research documents, whether they are published or not. The documents may come from teaching and research institutions in France or abroad, or from public or private research centers.
L'archive ouverte pluridisciplinaire HAL, est destinée au dépôt et à la diffusion de documents scientifiques de niveau recherche, publiés ou non, émanant des établissements d'enseignement et de recherche français ou étrangers, des laboratoires publics ou privés. 


\title{
ALMA, A Logistic Mobile Application based on Internet of Things
}

\author{
Didier El-Baz*, Julien Bourgeois ${ }^{1}$, Toufik Saadi², Alessandro Bassi ${ }^{3}$ \\ *CNRS, LAAS, 7 avenue du colonel Roche, F-31400 Toulouse, France \\ Université de Toulouse, LAAS ; F-31400 Toulouse France \\ e-mail : elbaz@laas.fr \\ ${ }^{1}$ Université de Franche-Comté - FEMTO-ST Institute, UMR CNRS 6174 \\ 1 cours Leprince-Ringuet ; F-25200 Montbéliard France \\ ${ }^{2}$ EPROAD Lab \\ Université de Picardie, 33 rue Saint Leu ; F-80039 Amiens Cedex 1, France \\ ${ }^{3}$ Alessandro Bassi Consulting, \\ 3 avenue de Cannes, F-06160 Juan les Pins, France
}

\begin{abstract}
A Logistic Mobile Application is presented. The application is based on Internet of Things and combines a communication infrastructure and a High Performance Computing infrastructure in order to deliver mobile logistic services with high quality of service and adaptation to the dynamic nature of logistic operations.
\end{abstract}

Keywords-component; Internet of Things; logistics; combinatorial optimization; high performance computing

\section{INTRODUCTION}

Logistic operators deliver goods to customers; the optimization of quality of service, e.g., on-time delivery and cost delivery is of major concern in this domain; this necessitates the optimization of truck loading and vehicle routing. The nature of logistic applications is dynamic, e.g., good delivery orders or cancellations may occur at any time; transportation difficulties may also occur at any time; these vicissitudes may be due to vehicle faults, traffic jam or particular weather conditions.

A Logistic Mobile Application (ALMA) proposes a mobile, real time, IoT-based solution in order to take into account the dynamic nature of logistic problems and to optimize the quality of service. Mobile devices like smart phones are used to report good delivery occurrences and incidents like an engine fault or a traffic jam; they are also used in order to launch computations related to the solution of a resulting routing problem on computing infrastructures in order to cope with incidents in real time. The ALMA project relies on a new High Performance Computing (HPC) infrastructure that makes use of clusters, grids and peer-to-peer networks via a broker that takes into account computational need and machines availability. We note that the peer-to-peer concept has known great developments with file sharing applications like Gnutella [1] or FreeNet [2]. Recent advances in microprocessors architectures, e.g., the generalization of the concept of parallelism and advances in high bandwidth networks permit one to consider high performance peer-to-peer computing as an economic and attractive solution. The ALMA project relies also on new optimization algorithms for the solution of combined truck loading and vehicle routing problems.

\footnotetext{
"Part of this work was funded by ANR.
}

Section II deals with logistics. We present the global ALMA architecture in section III. In particular, we detail the communication infrastructure and the HPC infrastructure. A first series of computational results is presented in Section IV. Finally, conclusions and future work are treated in section V.

\section{LoGISTICS}

Logistic applications are difficult problems; most of them are NP-complete problems (see [3] to [7]). The ALMA logistic application considers combined truck loading and vehicle routing problems. Treatment of vehicle routing problems in conjunction with truck loading is very attractive in just-in-time distribution context; indeed the stock can be close to zero (no stock). This technique is used more and more in mass-market retailing and car manufacturing. In spite of the advantages of just-in-time distribution and in particular its influence on cost reduction; it may generate weakness in the logistic chain in case of failure; hence the necessity to treat dynamically and as quickly as possible the events that may perturb the correct working of the logistic chains.

Treatment of vehicle routing problems in conjunction with truck loading has been studied in the literature (see [3] to [6]). The ALMA logistic application concentrates also on dynamic logistic problems whereby dynamicity results from new orders, cancellations, as well as traffic incidents that may occur at any time; this leads to extremely difficult problems. Our approach is essentially based on the approximate solution of truck loading problems via strip generation and beam search (see [7] to [9]); vehicle routing problems are solved via Ant Colony Optimization (ACO) [10]. The approach relies on parallel and distributed computing via several types of architectures, e.g., clusters, grids or peer-to-peer infrastructures since those problems are difficult to solve.

\section{Global Alma Architecture}

The ALMA logistic application relies on two infrastructures: a communication infrastructure and a HPC infrastructure. Fig. 1 displays the infrastructures of the mobile application ALMA.

\section{A. The communication infrastructure}

Goods to be delivered are identified by tags. When a good is delivered, the transporter scans the tag and transmits the 
information in real time to the logistic centre with a smart phone connected to the Internet via a $3 \mathrm{G}$ connection. The mobile application is based on the existing telecommunication infrastructure. Similarly, the transporter informs the centre in real time of traffic incidents, like road closed and traffic jam. In case of problems, e.g. traffic incidents, the proposed initial route may not be valid. Thus the transporter uses also the mobile application to ask for a new route. The request for a new route is transmitted to the broker of the HPC infrastructure.

\section{B. The HPC infrastructure}

\section{1) The broker}

The broker is designed in order to select a convenient HPC infrastructure among several available parallel or distributed architectures. These architectures may be clusters, grids or peer-to-peer networks. For a given instance of vehicle routing problem and method, the broker selects also a convenient topology and number of machines. This represents an evolution in comparison with the approach presented in [11]. The main goal of the broker is to select a computing infrastructure that satisfies the real time constraints of the application. Vehicle routing solution requests are associated with a deadline for result reception so as to limit vehicle idle time since computation time that are too long lead to a blocking of the logistic application. We note that if the infrastructure selection is not convenient, then a suboptimal solution far from the optimum will not be appropriate.

Two main phases can be considered for brokering: first, the supervision of available resources, e.g. clusters, grids or peerto-peer networks. Secondly, the prediction of computation time for the considered problem and selected method. We note that these steps can be iterated several times in order to improve prediction. Reference is made to [12] to [14] for previous work on performance prediction of HPC applications on distributed computing infrastructures.

\section{2) The environment for computing}

The environment for computing is an extension of P2PDC (see [11]). Reference is also made to [15] and [16] for more details and extensions of P2PDC. We recall that P2PDC was originally designed as a decentralized environment for peer-topeer High Performance Computing; P2PDC is particularly devoted to task parallel applications. P2PDC is intended in particular to scientists who want to solve numerical simulation problems or optimization problems via distributed iterative methods that lead to frequent direct data exchanges between peers. P2PDC relies on the use of the P2PSAP self adaptive communication protocol [17] (see Fig. 2) and a reduced set of communication operations (P2Psend, P2Preceive and $\mathrm{P} 2 \mathrm{P}$ wait) in order to facilitate programming. The programmer cares only about the choice of distributed iterative scheme of computation (synchronous or asynchronous) that he wants to be implemented and does not care about the communication mode between any two machines. The programmer has also the possibility to select a hybrid iterative scheme of computation, whereby computations are locally synchronous and asynchronous at the global level.

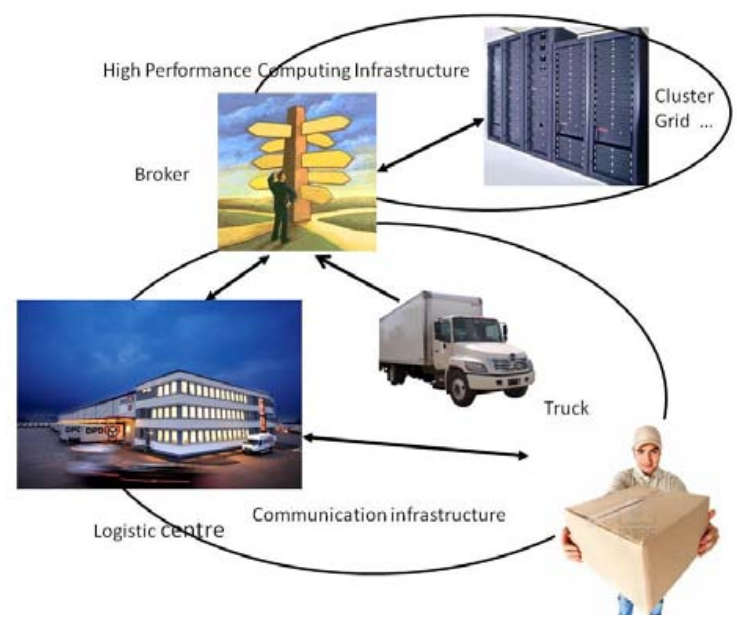

Figure 1. Communication and HPC infrastructures of the mobile application ALMA (graphics : various Internet sources).

P2PSAP chooses dynamically the most appropriate communication mode between any two peers according to a decision taken at application level like scheme of computation and elements of context like network topology at transport level. In the hybrid case, the communication mode between peers in a group of machines that are close and that present the same characteristics is synchronous and the communication mode between peers in different groups is asynchronous. The decentralized environment P2PDC is based on a hybrid topology manager and a hierarchical task allocation mechanism which make P2PDC scalable. We note that the P2PSAP communication protocol was designed first as an extension of the CTP transport protocol [18] based on the CACTUS framework which uses the concept of microprotocols (see [19]).

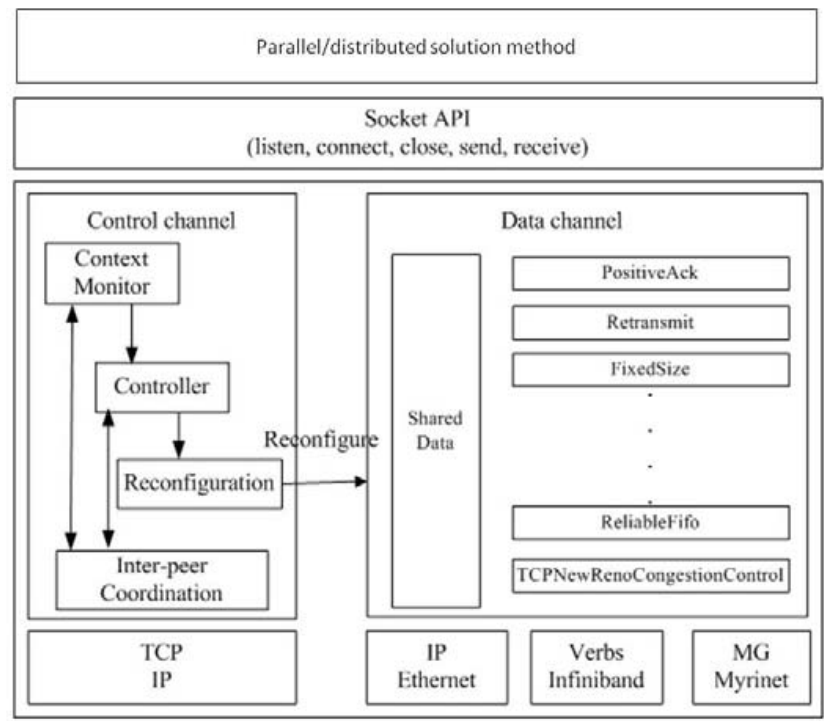

Figure 2. P2PSAP protocol architecture. 
The CTP protocol includes a wide range of micro-protocols including a small set of basic micro-protocols like Transport Driver, Fixed Size or Resize and Checksum that are needed in every configuration and a set of micro-protocols implementing various transport properties like acknowledgements, retransmissions, error correction and congestion control. The P2PSAP communication protocol takes into account Ethernet and Infiniband clusters. Reference is also made to [20] for details on peer-to-peer computing.

\section{EXPERIMENTAL RESULTS}

We consider here loading problems and present preliminary experimental results obtained for a $2 \mathrm{D}$ cutting stock problem solved with a two-stage two-dimensional method based on strip generation and beam search via the decentralized environment P2PDC on the Grid 5000 testbed. Reference is made to [21] and [22] for details on the two-stage two-dimensional method based on strip generation and beam search.

Fig. 3 displays the number of active processors during the solution of a cutting stock problem in function of the time. A maximum of twenty processors were allocated to this particular problem. We note that the number of active processors varies according to the evolution of the algorithm, i.e. the need of computing resources to treat in parallel the problem. In the beginning, the solution needs few computing resources since the number of nodes to explore is small. The number of processors increases with the time (since more and more nodes to explore are created) till the limit is met, i.e., the maximum number of twenty processors that were allocated to the solution of this problem. Finally, the number of active processors decreases since the number of nodes to explore decreases.

Obtaining a good approximation of the best solution at a given processor and communicating it to other processors permits one sometimes to decrease dramatically the need for computing resources. This is what we observe when the number of processors decreases suddenly from twenty to fifteen. Nevertheless, we observe that the number of needed computing resources may increase for a while before finally tending to zero at the end of the computation.

Fig. 4 displays solution times for several instances of cutting stock problems according to the maximum number of allocated processors.

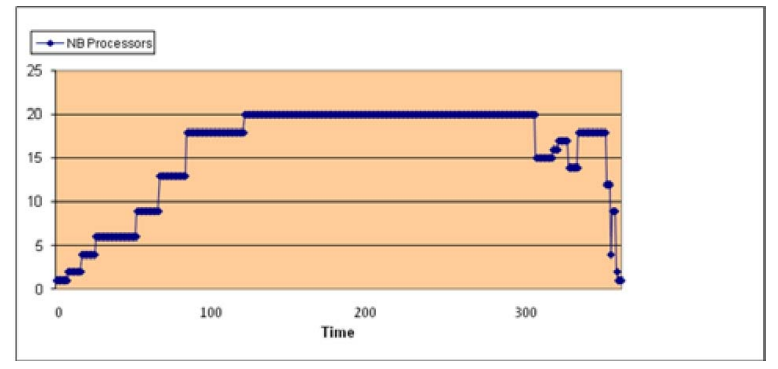

Figure 3. Cutting stock problem, number of active machines.

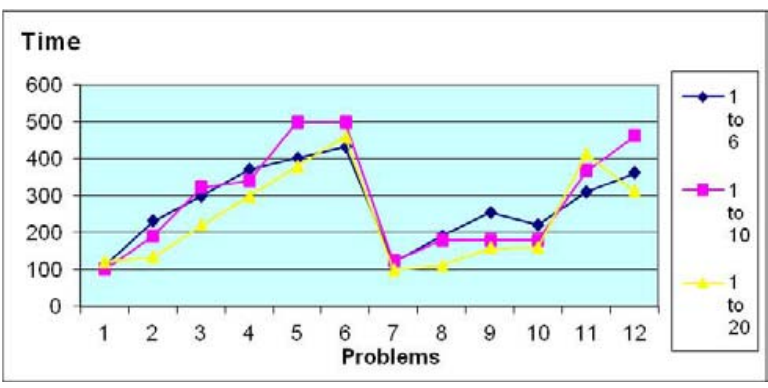

Figure 4. Cutting stock problem, solution time according to the maximum number of allocated machines.

Three cases are considered: a case with a maximum number of six machines (diamonds), a case with maximum number of ten machines (squares) and finally a case with twenty machines (triangles). We note that in general, the more processors are allocated, the smaller the solution time. Reference is made to [23] and [24] for details on peer-to-peer distributed algorithms for 2D Cutting stock problems. The reader is also referred to [25] for distributed branch and bound on peer-to-peer networks.

\section{Conclusions}

In this paper, we have presented the logistic mobile application ALMA that is based on the Internet of Things. ALMA addresses dynamic logistic problems whereby new orders or cancellation, as well as traffic incidents may occur at any time. The ALMA application permits one to communicate in real time the information regarding delivery of goods.

The logistic application ALMA combines a communication infrastructure and a parallel / distributed computing infrastructure in order to obtain rapidly new routes for transporters that deliver goods to customers in case of incidents like traffic jam. The HPC infrastructure makes use of a broker to select the convenient parallel / distributed architecture as well as the number of computing resources to perform computations according to a fixed deadline. Clusters, grids or peer-to-peer infrastructures can be selected among a pool of available parallel / distributed infrastructures. The computing infrastructure makes use of the High Performance Computing decentralized environment P2PDC in order to facilitate its use.

The mobile application ALMA addresses also combined truck loading and vehicle routing problems that lead to very complex optimization problems. A first series of computational experiments for cutting stock problems solved on Grid 5000 has been presented and analyzed in the paper. In particular, this permits one to illustrate the interest of the proposed approach.

We are presently extending the decentralized environment P2PDC for high performance distributed computing to Myrinet clusters and infrastructures combining Infiniband, Myrinet and Ethernet networks. We plan also to extend the P2PDC environment to heterogeneous architectures combining multicore CPUs and GPUs. Self organization of machines for 
deployment and efficiency purposes or for insuring everlastingness of applications in hazardous situations or in the presence of faults will be also studied.

\section{ACKNOWLEDGMENT}

The authors wish to thank Agence Nationale de la Recherche (ANR) for support through several contracts. Experiments presented in this paper were carried out using the Grid' 5000 experimental testbed being developed under the INRIA ALADDIN development action with support from CNRS, RENATER and several Universities as well as other funding bodies.

\section{REFERENCES}

[1] Gnutella Protocol Development. http://rfc-gnutella.sourceforge.net.

[2] The FreeNet Network Projet. http://freenet.sourceforge.net.

[3] M. Iori, J. J. Salazar Gonzalez, D. Vigo, "An exact approach for the symmetric capacited vehicule routing problem with two dimensional loading constraints". Technical Report OR/03/04, DEIS, Universita de Bologna, 2003.

[4] M. Gendreau, M. Iori, G. Laporte, S. Martello, "A Tabu search heuristic for the vehicle routing problem with two-dimensional loading constraint”, Management Science, Vol. 40, $\mathrm{N}^{\circ} 10,1994$, pp. 12761290.

[5] E. Choi, D. W. Tcha, "A column generation approach to the heterogeneous fleet vehicle routing problem", Computers and Operations Research, Vol. 34, 2007, pp. 2080-2095.

[6] K. Ganesh, T. T. Narendran, "CLOVES: a cluster-and-search heuristic to solve the vehicle routing problem with delivery and pick-up", European Journal of Operational Research, Vol. 178, 2007, pp. 699717.

[7] M. Hifi, R. M'Hallah et T. Saadi, "Approximate and exact algorithms for the double constrained two-dimensional guillotine cutting stock problem", Computational Optimization and Applications, 2007, pp. 2873- 2894.

[8] M. Hifi, R. MHallah T. Saadi, "Algorithms for the constrained twostaged two-dimensional cutting problem", INFORMS Journal on Computing, Vol.20, 2008, pp.212-221.

[9] M. Hifi, T. Saadi, "A cooperative algorithm for constrained two-staged two-dimensional cutting problems", International Journal of Operational Research, Vol. 9, No.1, 2010, pp. 104-124.

[10] M. Dorigo, "Optimization, Learning and Natural Algorithms", $\mathrm{PhD}$ thesis, Politecnico di Milano, Italy, 1992.

[11] T. T. Nguyen, D. El Baz, P. Spiteri, J. Jourjon, M. Chau, "High performance peer-to-peer distributed computing with application to obstacle problem", Proc. $24^{\text {th }}$ IEEE International Parallel \& Distributed Processing Symposium and Workshops (IPDPSW), Atlanta USA, May 2010, pp. 1453-1461.

[12] B. Cornea, J. Bourgeois, T. T. Nguyen, D. El Baz, "Performance prediction in a decentralized environment for peer to peer computing", Proc. $25^{\text {th }}$ IEEE International Parallel \& Distributed Processing Symposium and Workshops (IPDPSW), Anchorage USA, May 2011, pp. 1613-1621.

[13] B. Cornea, J. Bourgeois, "Performance prediction of distributed applications using block benchmarking methods", Proc. $19^{\text {th }}$ Conference on Parallel, Distributed and networked-based Processing, PDP 2011, Ayia, Napa Cyprus, Feb. 2011, pp. 183-190.

[14] B. Cornea, J. Bourgeois, "A framework for efficient performance prediction of distributed applications in heterogeneous systems", The Journal on Supercomputing, 2012, pp. 1609-1634.

[15] B. Cornea, J. Bourgeois, T.T. Nguyen, D. El Baz, "Scalable performance predictions of distributed peer-to-peer application", Proc. $14^{\text {th }}$ IEEE International Conference on High Performance Computing and Communication, Liverpool U.K, 2012, pp. 193-201.

[16] [31] T. Garcia, M. Chau, T. T. Nguyen, D. El Baz, P. Spiteri, "Asynchronous peer-to-peer distributed computing for financial applications", in Proc. $25^{\text {th }}$ IEEE International Parallel \& Distributed
Processing Symposium and Workshops (IPDPSW), Anchorage USA, May 2011, pp. 1453-1461.

[17] D. El Baz, T. T. Nguyen, "A self-adaptive communication protocol with application to high performance peer-to-peer distributed computing", Proc. of the $18^{\text {th }}$ Conference on Parallel, Distributed and networked-based Processing, PDP 2010, Pisa Italy, Feb. 2010, pp. 327333.

[18] G.T Wong, M.A Hiltunen, R.D Schlichting, "A configurable and extensible transport protocol”, Proc. IEEE INFOCOM'01, Anchorage, Alaska USA, 2001, pp. 319-328.

[19] M. A. Hiltunen, "The Cactus approach to building configurable middleware services", Proc. DSMGC2000, Nuremberg Germany, 2000

[20] D. El Baz, G. Jourjon, "Some solutions for Peer to Peer Global Computing", Proc. $13^{\text {th }}$ Conference on Parallel, Distributed and networked-based Processing, PDP 2005, Lugano Switzerland, Feb. 2005, pp. 49-58.

[21] M. Hifi, R. M'Hallah, "An exact algorithm for constrained twodimensional two-staged cutting stock problems", Operations Research, Vol. 53, 2005, pp. 140-150.

[22] M. Hifi, T. Saadi, "A parallel algorithm for constrained two-staged two-dimensional cutting problems", Computers and Industrial Engineering, Vol. 62, $\mathrm{N}^{\circ} 1,2012$, pp. 177-189.

[23] M. Hifi, T. Saadi, N. Haddadou, "High performance peer-to-peer distributed computing with application to constrained two-dimensional guillotine cutting problem", Proc. $19^{\text {th }}$ Conference on Parallel, Distributed and networked-based Processing, PDP 2011, Ayia Napa Cyprus, Feb. 2011, pp. 552-559

[24] D. El Baz, M. Hifi, T. Saadi, "Peer-to-peer solution of 2D-cutting stock problems, Proc. $11^{\text {th }}$ Cologne-Twente Workshop on Graphs and Combinatorial Optimization, Munich Germany, May 2012, pp. 116-120.

[25] M. Djamaï, B. Derbel and N. Melab, "Distributed B\&B: a pure peer-topeer approach", Proc. $25^{\text {th }}$ IEEE International Parallel \& Distributed Processing Symposium and Workshops (IPDPSW), Anchorage USA, May 2011, pp. 1788-1797. 